\title{
Differentiation of Thoracic Outlet Syndrome from Treatment-Resistant Cervical Brachial Pain Syndromes: Development and Utilization of a Questionnaire, Clinical Examination and Ultrasound Evaluation
}

Sheldon E. Jordan, MD', Samuel S. Ahn, MD², and Hugh A. Gelabert, MD²

From: ${ }^{1}$ Neurological Associates of West Los Angeles, Santa Monica, CA and ${ }^{2} U C L A$ Department of Vascular Surgery, Los Angeles, CA.

Dr. Jordan ${ }^{1}$ is Clinical Associate Professor of Neurology with the UCLA Department of Neurology and

Saint John's Health Center, John

Wayne Institute, Santa Monica, CA. Dr. Ahn ${ }^{2}$ is Attending Surgeon, UCLA

School of Medicine, Department of Surgery, Division of Vascular Surgery,

and with University of Vascular

Associates. Los Angeles, CA. Dr.

Gelabert ${ }^{3}$ is Professor of Surgery,

Department of Surgery, Division of Vascular Surgery, UCLA School of Medicine, Los Angeles, CA.

Address Correspondence: Sheldon E Jordan, MD

Neurological Associates of West Los Angeles

2811 Wilshire Blvd, Suite 790

Santa Monica, California 90403

E-mail: shellyj@aol.com Funding: None.

Conflict of Interest: None.

Manuscript received on: 03/12/2007

Revisions accepted: 04/08/2007 Accepted for publication on:

04/24/2007

Free Full manuscript www.painphysicianjournal.com
Objective: The present study was undertaken to determine which factors differentiate patients with a good outcome after treatment for Thoracic Outlet Syndrome (TOS) from patients with a poor outcome.

Methods: A total of 85 patients, who were examined during one year, had at least 6 months of follow up after treatment for TOS with either surgery or botulinum chemodenervation.

Results: Socioeconomic factors of work disability or workers' compensation claims did not differentiate treatment-responsive TOS from treatment-resistant cases. There was no difference between the 2 groups regarding the presence of anomalous anatomy detected by ultrasonography or regarding the presence of subclavian artery flow acceleration or occlusion detected by duplex sonography. Several factors were noted more frequently in treatment-resistant patients: sensory complaints extending beyond lower trunk dermatomes ( $42 \%$ vs. $10 \%)$, weakness extending beyond lower trunk myotomes $(19 \%$ vs. $2 \%)$, histories of previous non-TOS surgery of the neck or upper limbs $(50 \%$ vs. $17 \%)$, comorbidities of fibromyalgia or complex regional pain syndrome $(81 \%$ vs. $12 \%$ ), and depression (35\% vs. 10\%). Treatment-resistant patients complained about more widespread functional impairments on a validated Cervical Brachial Symptom Questionnaire (CBSQ) than treatment-responsive patients. Resistant cases responded less often to a scalene test block (38\% vs. $100 \%$ ), which is designed to simulate the effects of targeted treatment.

Conclusion: In summary, compared to patients with a good outcome after targeted treatment, patients with a poor outcome had more diffuse complaints and responded less often to a scalene test block.

Keywords: Cervical brachial pain, chemodenervation, thoracic outlet syndrome, ultrasound

Pain Physician 2007; 10:441-452 
ervical brachial pain, pain in the neck, arm and shoulder, is a frequent complaint of patients presenting to pain medicine practices. Specific injection and surgical techniques may be considered for certain diagnoses including Carpal Tunnel Syndrome, Ulnar Neuropathy, Thoracic Outlet Syndrome (TOS) and Cervical Radiculopathy. In order to maximize the chances of a successful outcome, it is important to identify factors that would help determine if a patient is likely to benefit from an intervention. The present paper was offered to determine which factors may be considered in selecting patients with TOS who may benefit from specific treatment.

When exercises and ergonomic adjustments fail, patients with TOS may be successfully treated with specifically targeted approaches such as surgery or botulinum chemodenervation (1-10). The various surgical approaches and botulinum chemodenervation share a common feature; they are designed to decompress the thoracic outlet so that clinical improvement should reflect an improvement in symptoms and functionality resulting from decompression of the vascular and neurological elements. Although placebo effects and other non-specific factors may detract from the analysis, improvement after application of either would not be expected unless the patient actually had TOS. Improvement after either treatment may be considered one way of retrospectively confirming a case of "true" TOS. After accumulating a group of such treatment successes it should be possible to contrast such cases with a group of treatment failures so that it would be possible to identify factors which may be used to differentiate the treatment-responsive cases from treatment resistant cases. The present study was undertaken to identify which factors differentiate cases of treatmentresponsive TOS from cases with treatment-resistant cervical brachial pain.

Although a matter of some controversy, certain socioeconomic factors are often considered as contributing to treatment resistance in cases with chronic pain; these factors include the presence of ongoing workers' compensation claims, disability status and a history of personal injury litigation (11-25) In designing this study it was expected that socioeconomic factors would be associated with treatment resistant cases of cervical brachial pain. Psychological factors have also contributed to treatment failure in other studies; it would be expected that the presence of depression would be associated with treatment resistance in TOS, as well $(26,27)$
Individual clinical features such as ultrasound evidence of positional arterial occlusion, the presence of interscalene triangle anomalies on ultrasound and the results of diagnostic "scalene blocks" have also been used to diagnose patients with potentially treatable TOS (28-33). It would be expected that certain comorbid factors such as the presence of more widespread neuropathic complaints or the presence of chronic pain syndromes such as fibromyalgia and CRPS would be associated with treatment resistant cases (34). Furthermore, the performance on symptom questionnaires, pain diagrams and muscle testing has been used to diagnose patients with specific cervical brachial pain syndromes (36-38). It was expected that patients with TOS should not demonstrate an overabundance of symptoms when responding to the symptom surveys. These patients should also demonstrate patterns of sensory disturbance and motor weakness that more often localizes to the lower trunk of the brachial plexus rather than to more widespread levels of the neuraxis. How the latter clinical factors differentiate treatmentresponsive TOS from treatment-resistant cases was also the focus of this study.

\section{Methods}

The study protocol and consent process was approved by the Institutional Review Board for the protection of human subjects. Initially, a Cervical Brachial Symptom Questionnaire (CBSQ) (Appendix 1) was developed and tested for reliability and validity in a sample of patients presenting with cervical brachial complaints and being evaluated for TOS at UCLA which has established a tertiary referral practice for the evaluation and treatment of patients with TOS so that 85 patients could be entered into this study according to criteria outlined. The numbers of subjects used for the various phases of reliability and validity testing are noted in the results section. The CBSQ questionnaire was based on a structured interview that had been used over the past 25 years in our practice that included 14 questions and a symptom diagram. The subjects were asked to scale how often the performance of certain activities resulted in symptoms that were severe enough to result in the cessation or modification of the activities. Each question had a numerical value of 0 to 10 and scoring consisted of a simple addition of all line item scores. In addition, a pain map and sensory disturbance diagram was filled out by each patient as part of the CBSQ. Patients were asked to label the diagram for areas of pain and also 
for areas of sensory change including "numbness" or "other sensory change including tingling." Content validity of the CBSQ was determined upon review by 2 vascular surgeons and 3 neurologists. Concurrent validity was determined by comparison with the Brief Pain Inventory. Test-retest reliability was determined by correlating full scale scores in tests that were performed less than one month apart in subjects that were clinically unchanged. Internal consistency was determined by calculating Cronbach's alpha. Responsiveness was determined by comparing questionnaires from patients in a baseline state versus questionnaires from patients after targeted treatment, which included either botulinum chemodenervation or surgery for TOS; the standardized response mean and the effect size were calculated.

Every patient seen within one calendar year was entered into the study if the patient was followed up for at least 6 months after a targeted treatment for TOS, consisting of either botulinum chemodenervation or TOS consisting of a first rib resection. The protocol for botulinum chemodenervation has been previously described (10). Two changes to the chemodenervation protocol are 1) an expanded muscle target set to include the subclavius and pectoralis minor muscles along with the previously targeted anterior scalene and middle scalene and 2) the use of intraoperative ultrasound for targeting rather than fluoroscopic guidance. Using a 7.5 to 10 megahertz ultrasound probe each targeted muscle was individually imaged in transverse or longitudinal plane. A 25-gauge needle, which was insulated except at the tip, was placed under continuous ultrasound and electromyographic monitoring into each target muscle. Botulinum type $A$ was injected with the following fixed doses: anterior scalene 10 units, middle scalene 12 units, subclavius 20 units, and pectoralis minor 35 units.

Each patient presenting with a cervical brachial pain syndrome and possible TOS was examined clinically for pulse obliteration in 90-degree and 130degree abduction, for Tinel's sign over the brachial plexus, over the ulnar nerve at the elbow, and over the median nerve at the wrist. A one minute elevated arm stress test was performed and subjects rated total discomfort (including pain, fatigue and dysesthesia or numbness) on a 0 to 10 numerical scale. After the one minute elevated arm stress test, all patients had a "scalene test block" according to a previously published protocol. Twenty-five gauge needles, insulated except at the tips, were inserted into the ante- rior scalene, subclavius, pectoralis minor and at least two control muscles including the sternocleidomastoid and trapezius muscles. Concordant or discordant pain was noted after motor threshold level stimulation of the motor point at each target with acceptable stimulation levels at less than 2 milliamps. The patient was blinded from the identity of each stimulation site with hand signals between technician and physician. Injections of anesthetics were performed in a double blinded fashion with either Lidocaine $2 \%$ or Marcaine $.75 \%$ for a total of one $\mathrm{mL}$ of injectant into the anterior scalene and $1.5 \mathrm{~mL}$ into the subclavius and pectoralis minor. Control muscles were injected with saline. Immediately after injection and every hour thereafter for nine hours, a pain diary was filled out by the patient noting total discomfort at rest and after a one minute elevated arm stress test as noted above (33). The only change in the protocol is that 1) additional targets include the subclavius and pectoralis minor in addition to the originally targeted anterior scalene and 2) intraoperative ultrasound is used for imaging guidance rather than fluoroscopy. A duplex ultrasound was performed for the subclavian artery in an infraclavicular window in neutral position and in 90 and 130 degrees of abduction. A 7.5 to 10 megahertz probe was used to evaluate anatomical images of the interscalene triangle, the infraclavicular space, the cubital tunnel, and the carpal tunnel. The presence of any extra structure between the lower trunk of the plexus and the first rib or any extra muscle tissue between the middle and lower plexus and subclavian artery was noted as an "anomaly" whether or not there was any obvious displacement or compression of the neurovascular elements.

The diagnosis of TOS was made if a subject had a history of positional pain or paresthesias induced with overhead activity, which was reproduced in a concordant fashion with a one minute elevated arm stress test. Patients who appeared to have a clinical presentation more characteristic of another peripheral compression neuropathy or a cervical radiculopathy were not included in this study. In addition to TOS, patients were diagnosed with concomitant CRPS if there was allodynia, hyperalgesia as well as the presence of signs that may be attributed to sympathetic nervous system dysfunction including a prolonged periods of a red or blue hand, persistent swelling in the absence of venous occlusion, excessive warmth or sweating compared to the opposite hand. Transient pallor and coolness was not considered a sign of CRPS for the purposes 
of this study since these findings are common in patients with evidence of vascular compression and TOS. Concomitant Fibromyalgia was diagnosed according American College of Rheumatology guidelines (39). After interview, the presence of depression was determined if the patient fulfilled DSM-IV-TR criteria for a Major Depressive Episode, however, in contrast to DSM-IV-TR, no exclusion was made because of possible side effects of drugs or the effects of a medical condition (40).

\section{Results}

The CBSQ had a test-retest reliability of 0.87 (Spearman Rank Order Correlation test) in 50 patients who were able to be retested within one month and who were clinically unchanged. Internal consistency was 0.93 (Cronbach's alpha). In 140 patients, some of whom were not included in the final analysis because they did not have a full 6 months of follow-up completed as noted in the methods section, the correlation with the Brief Pain Inventory (total of pain and interference scales) was 0.70 (Spearman Rank Order Correlation test). Responsiveness to change as measured by the standardized response mean was 2.04 and the effect size was 1.53 when comparing patients before and after successful treatment, as determined by at least a 20 percent improvement in numerical pain scores on a 0 to 10 linear scale, improvement in $\mathrm{BPI}$ of at least 20 percent and the patient's overall verbalized self-assessment of improvement.

Eighty-five patients who had been prospectively followed in one calendar year had at least 6 months of observation after targeted treatment for TOS including $63(74 \%)$ women and $22(26 \%)$ men with a mean age of $41.1 \pm 10.8$ years. Twenty-three $(27 \%)$ of the cases were treated with surgery for TOS, the remainder $(73 \%)$ were treated with botulinum chemodenervation. Surgical treatment was independently determined by the surgical team at UCLA. Surgery was reserved for patients with a clinical diagnosis of TOS as defined above who had failed to improve according to patient satisfaction in terms of pain control and improvement in work and daily activities after more than one month of physical therapy, home exercises and correction of ergonomic factors. Twenty-nine (34\%) of the cases related their chronic pain condition to a personal injury. Sixty-two $(73 \%)$ of the cases had occupational or recreational exposures associated with prolonged and repetitive reaching, grasping and fingering activities which appeared to trigger symptoms. Thirty-nine patients $(46 \%)$ described using a computer keyboard and mouse for at least 5 hours per day. Two patients did not identify either a single traumatic event or a repetitive trauma as a factor in symptom causation. Thirty-four $(40 \%)$ cases had ongoing worker's compensation claims; none of these cases had settled their claims at the time of the treatment and throughout the follow up period. Forty-seven $(55 \%)$ were completely disabled. Twenty-three (27\%) cases had at least one surgery for a cervical brachial pain syndrome other than TOS.

Fifty-nine of the 85 (64\%) patients had a good outcome after botulinum chemodenervation or first rib resection for TOS as defined by a 50 percent decrease on a numerical pain scale, an improvement in daily activities and a decrease of medications for breakthrough pain when evaluated within 6 months after the targeted intervention and were therefore considered to have treatment-responsive TOS. The remaining 26 patients were considered to have treatment resistant cervical brachial pain for the purposes of this study. There were no differences between these 2 groups for disability status (29 of 59 vs. 18 of 26, respectively, Fisher exact $p=.1019,2$ tailed) or for the presence of ongoing worker's compensation claims ( 27 of 59 vs. 7 of 26, Fisher exact $p=.1492,2$ tailed).

All of the treatment-responsive TOS patients had a positive "scalene block." Only 10 of 26 (38\%) of the treatment resistant cases had a positive block; the remainder had a negative block. Ultrasound examination revealed transverse cervical bands or anomalous muscles in 45 of $56(80 \%)$ of the responsive cases and 17 of $26(65 \%)$ in the non-responsive cases. There was no difference between the 2 groups for the presence of flow acceleration more than $300 \mathrm{~cm} / \mathrm{sec}$ or occlusion by ultrasound in hyperabducted position (23 of 67 arms tested in treatment-responsive TOS vs. 10 of 29 arms tested in treatment-refractory patients; Fisher exact $p=1.00,2$ tailed).

Treatment-responsive TOS patients were much less likely to have a comorbid diagnosis of a more widespread pain syndrome; 7 of 59 (12\%) had a comorbid diagnosis of CRPS or fibromyalgia compared to 21 of $26(81 \%)$ cases with treatment-refractory cervical brachial pain (Fisher exact $p=.0000$, one tailed). Treatment-responsive TOS patients were less likely to have surgeries for conditions other than TOS (10 out of $59,17 \%$ vs. 13 out of $26,50 \%$, Fisher exact $p$ $=.0023$ ), less likely to have widespread sensory symptoms involving the upper arm and face ( 6 out of 59, $10 \%$ vs. 11 out of $26,42 \%$; Fisher exact $p=.0013$ ) and less likely to have weakness extending beyond the 
lower trunk myotomes (14 out of 118 hands, $12 \%$ vs. 15 out of $52,29 \%$, hands; Fisher exact $p=.0087$ ). There was a significant difference between the 2 groups for the presence of depression $(9 / 26,35 \%$ for the treatment resistant group vs. $6 / 59,10 \%$ for the treatmentresponsive group; Fisher exact $p=.0095)$. There was no difference between the groups on combined BPI pain and interference scales $(78.47 \pm 25.01$ vs. 79.19 \pm 16.22), however, treatment-responsive TOS patients as a group scored lower on the CBSQ than treatmentrefractory patients $(78.47 \pm 25.01$ vs. $94.15 \pm 17.51$; Mann Whitney $U$ test $p=.039490)$.

Combining the two of the above factors, it should be pointed out that 24 out of $26(92 \%)$ cases with refractory cervical brachial pain had either a negative scalene block or a comorbid diagnois of a widespread pain syndrome compared with 7 out of $59(12 \%)$ cases with treatment-responsive TOS (Fisher exact $p=.0000$, one tailed).

\section{Discussion}

The present study demonstrated that 2 socioeconomic factors did not differentiate treatment-responsive cases from those resistant to treatment, including the presence of a worker's compensation claim, or an ongoing disability unemployment status. Consistent with these results is the finding that both groups scored similarly on combined pain and interference scores on the BPI. The interference scores reflect the patients own assessment of overall disability for daily activities including work related activities. We had suspected that there might have been differences between groups in these socioeconomic factors; these same socioeconomic factors have been previously studied with various degrees of correlation or no correlation being detected in relation to treatment outcomes for chronic pain patients(12-25).

In the present study, depression was observed more frequently in the group of treatment resistant patients. In previous studies of chronic pain treatments, the presence of depression was predictive of poor outcomes $(26,27)$.

The ability of the scalene test block to differentiate treatment-responsive cases from treatment-refractory cases was the subject of a previous report (33). The frequency of false negatives has been reduced compared to the prior study, probably as a result of adding more injection sites to the original single scalene target; combining the subclavius and pectoralis minor sites was expected to yield lower false negatives since there is a better chance of temporarily decompressing the costoclavicular space and sub pectoral space with the extended target set.

Besides the findings on the scalene block, the main findings can be summarized by pointing out that treatment-responsive TOS patients have more restricted and localized signs and symptoms than the treatment resistant patients. The former group had sensory complaints more often confined to the hand and forearm as opposed to more widespread sensory complaints involving the upper arm or face. When patients with treatment-responsive TOS have weakness, it is more likely to be confined to the lower trunk myotomes rather than involving more widespread levels of motor involvement. Patients with treatment-responsive TOS are also less likely to have had failed surgeries directed at non-TOS surgical targets; furthermore, these patients complain of fewer specific functional disturbances on a validated CBSQ symptom survey compared to treatment resistant patients.

Other factors, not explored in this survey may also predict treatment responsiveness in patients with TOS such as the presence of certain body postures, and the presence of certain body types including those with asthenia or obesity.

\section{Conclusions}

Based on the present study, it would appear that selection criteria for TOS surgery or chemodenervation should include the present iteration of the scalene block (scalene-subclavius-pectoralis minor block) and an exclusion for comorbid pain syndromes and for widespread sensory and motor findings that would indicate a problem that has extended beyond the more restricted neurological localizations typical for treatment-responsive TOS. Further research is needed to determine whether widespread or multifocal pain, including concomitant CRPS or fibromyalgia, would predict a poor outcome in other cervical brachial pain syndromes including radiculopathy, Carpal Tunnel Syndrome, and UInar Neuropathy. 
Appendix I: Cervical Brachial Symptom Questionnaire.

\title{
Cervical Brachial Symptom Questionnaire ("CBSQ")
}

\author{
NAME
} DATE

READ INSTRUCTIONS FIRST. This form is important for measuring the outcome of treatment. Based on your experiences in the PAST WEEK, answer the following questions regarding how often symptoms would be likely to increase if you were to engage in certain activities.

Circle the number corresponding to how likely it would be for symptoms to increase during an activity so much that you would have to stop or modify the activity.

\section{DO NOT LEAVE ANY BLANKS.}

If a CONSTANT ongoing symptom would not be more noticeable during the activity, mark the answer " 0 ."

If a symptom would increase during half of the instances of the activity, mark the answer "5."

Only mark "10" if your symptoms would increase during EVERY instance of the activity.

1. Pain going down the arm increases with neck movement, as in turning, flexing or extending the neck.

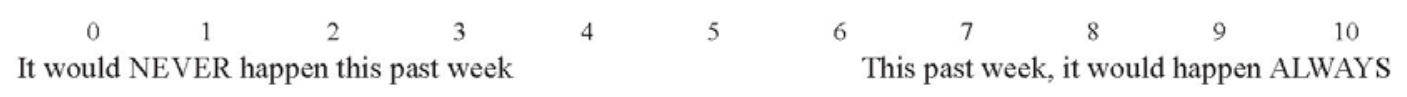

2. Pain in the arm or shoulder increases instantly with brief shoulder movement as in throwing something or in reaching behind the body.

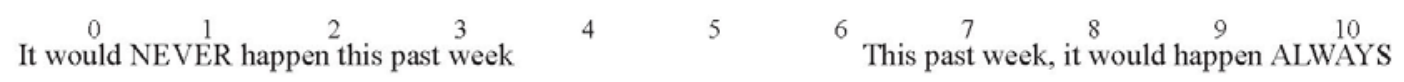

3. Hand or arm aches or fatigues with arm exercise, particularly with overhead or outstretched positioning.

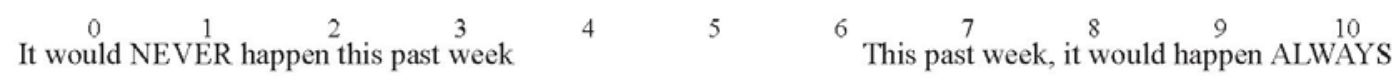

4. Hand or arm swells after arm exercise, including after any activities that require repetitive arm movements.

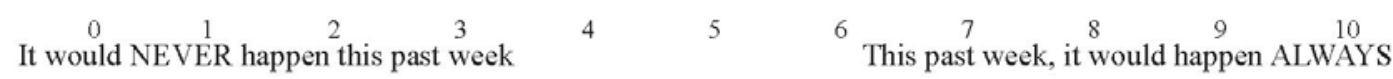

5. Sensations of tingling or numbness in the hand or arm increase when reaching overhead or outwards.

Examples include brushing hair or blow-drying hair, reaching for an overhead shelf, or working with arms overhead as in painting a ceiling or screwing in light bulbs.

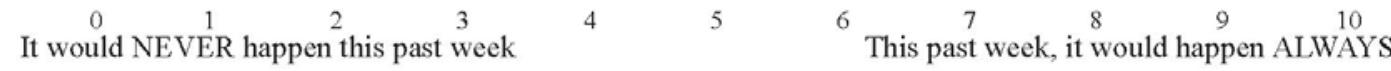

6. Sensations of tingling or numbness increase in the hand or arm when awakening from sleep.

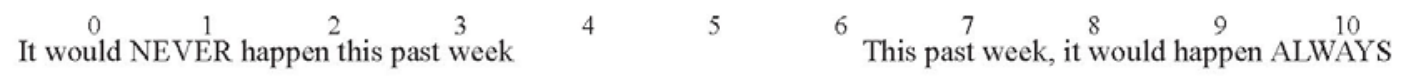


7. Sensations of tingling or numbness increase in the hand or arm with repetitive finger movements as in writing, typing, sewing, playing musical instruments or assembling objects.

$\begin{array}{lllllllllll}0 & 1 & 2 & 3 & 4 & 5 & 6 & 7 & 8 & 9 & 10\end{array}$

It would NEVER happen this past week This past week, it would happen ALWAYS

8. Sensations of tingling or numbness increase with prolonged or forceful grasping as in holding a steering wheel to drive, using tools, handling office instruments or controlling industrial equipment.

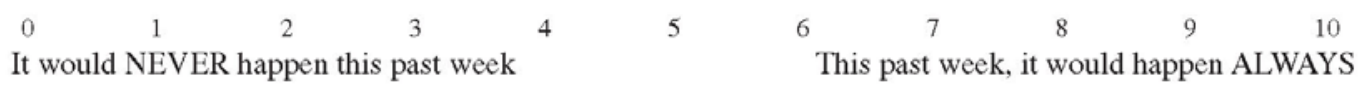

9. Sensations of tingling or numbness increase while bending elbow or leaning on elbow, for example, while holding telephone receiver or leaning on a desk.

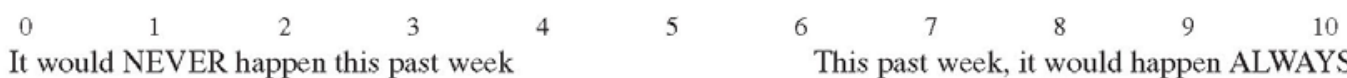

10. Hand is clumsy or weak while trying to hold onto objects or while attempting to open jars, use keys to open a lock, pull zippers or button clothing.

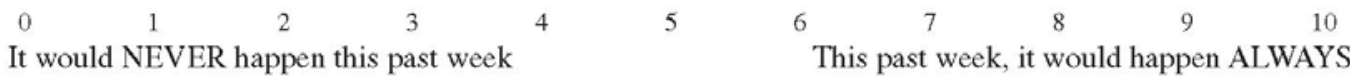

11. Pain is caused by experiences that ordinarily are not painful. Examples include a light touch to the hand, arm, or neck, such as a light draft, the rub and tug of clothing, or the touch of something moderately hot or cold.

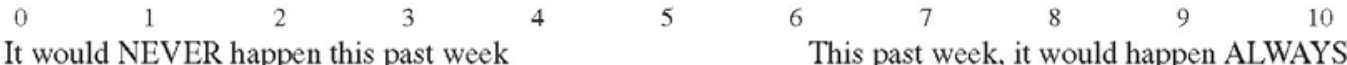

12. Disabling pain that can last into the next day is caused by activities that ordinarily produce only mild discomfort. Examples include a light exercise session, a physical therapy treatment or a physical examination.

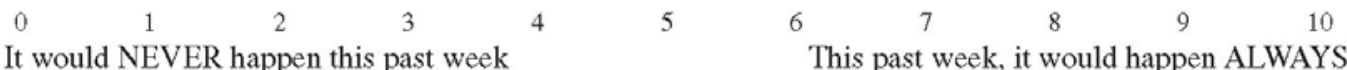

13. Symptoms have occurred with the above activities in the past without recurrence in the past week. yes no (circle your answer) If the answer is "yes", please list by number and explain on back.

14. Hand becomes blue, red, swollen, sweaty or hot. Yes No (circle answer) If "yes" explain on back. 


\section{CERVICAL BRACHIAL SYMPTOM QUESTIONNAIRE}

Mark where you feel pain with horizontal

with diagonal lines. If different pains or

sensory changes are caused by specific

items in the questionnaire, then indicate

by the question number.

Use next page if necessary. or vertical lines. Mark sensory changes

NAME

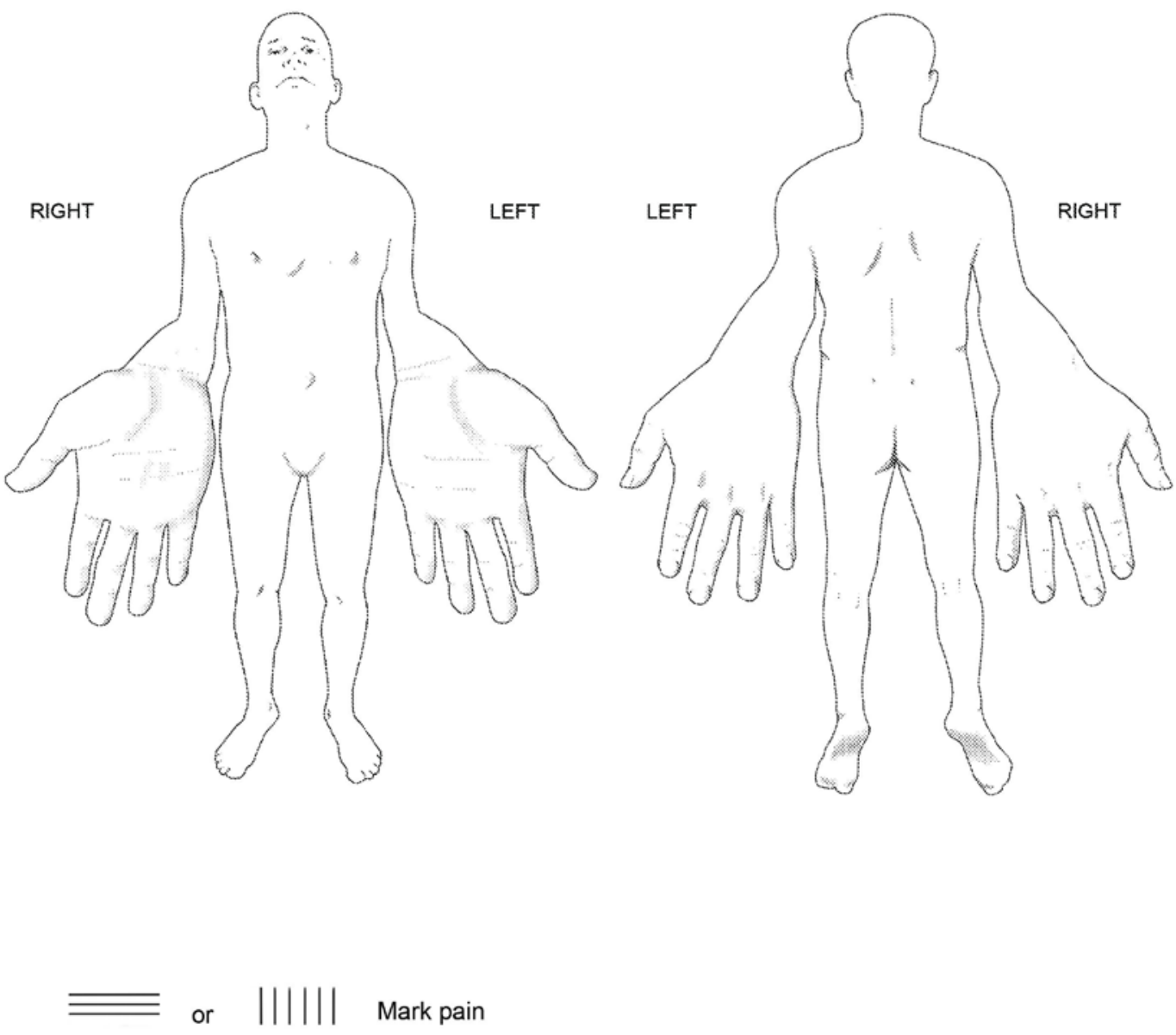

Mark numbness or sensory disturbance including tingling 


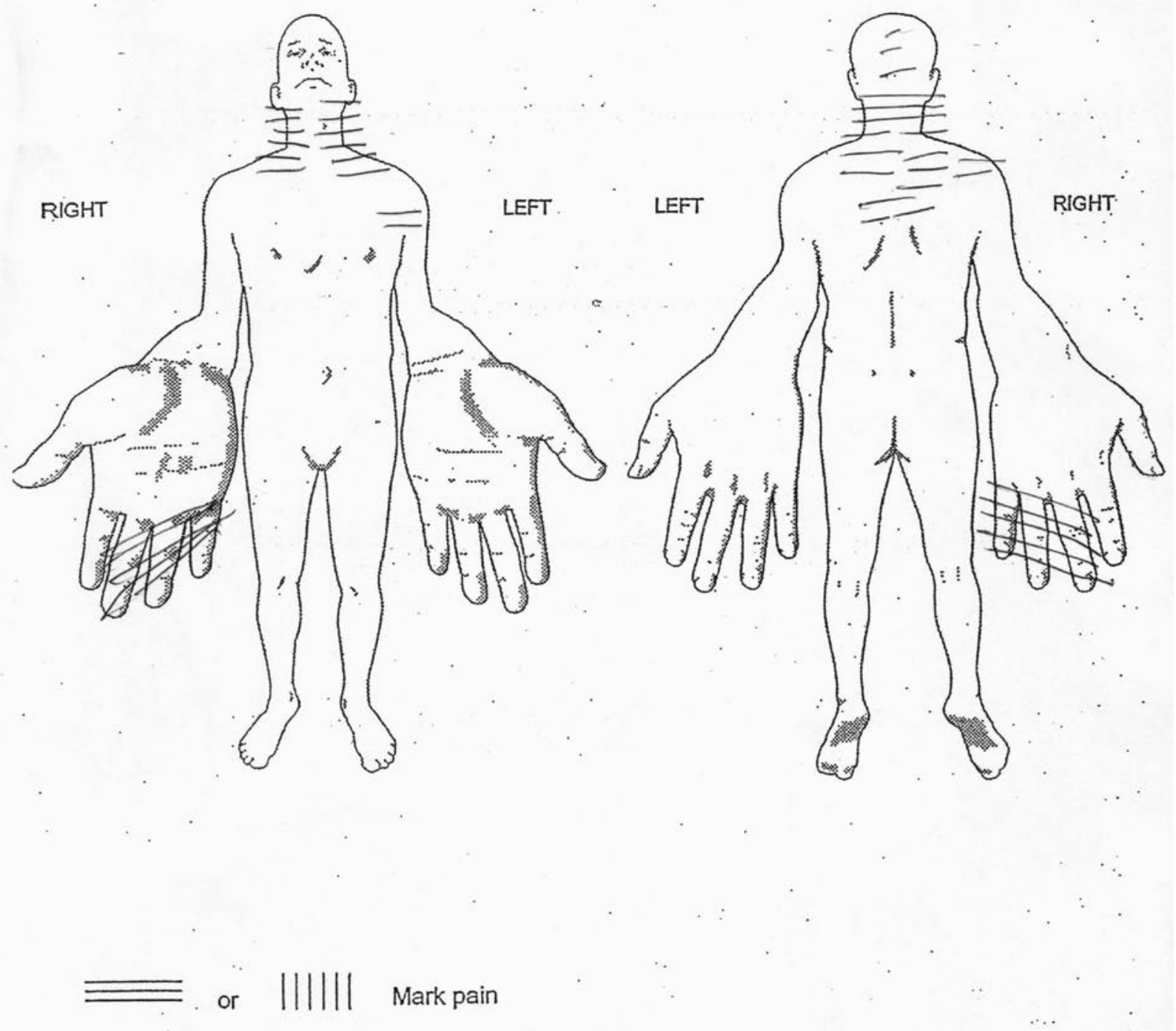

1111 or $I / / /$, Mark numbness or sensory disturbance including tingling

limited sensory disturbance in a patient with treatment responsive TOS 


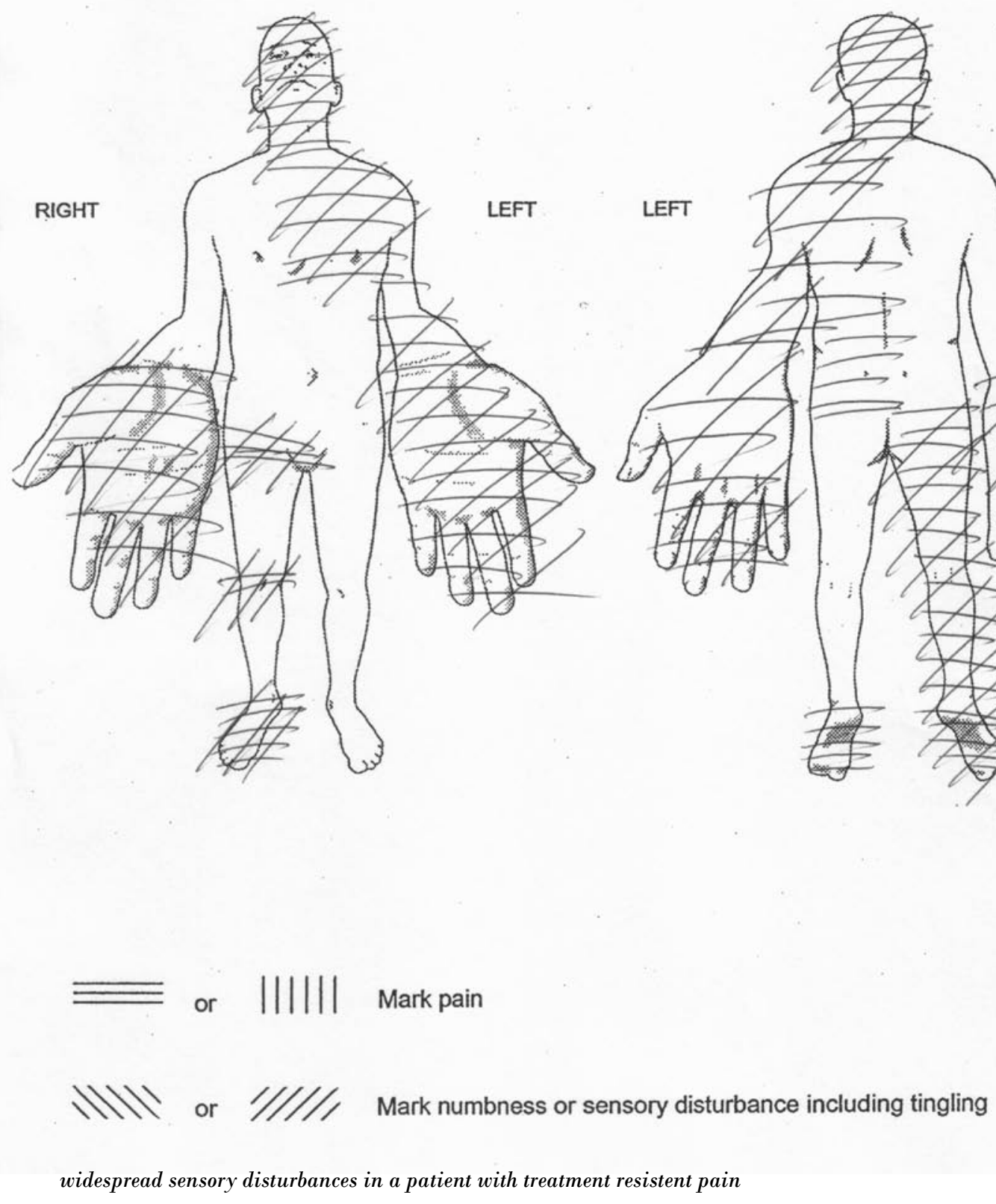




\section{References}

1. Sanders RJ, Hammond SL. Supraclavicular total scalenectomy with or without first rib resection: technique and results. Handchir Mikrochir Plast Chir 2006; 38:29-36.

2. Colli BO, Carlotti CG, Jr., Assirati JA, Jr., Marques W, Jr. Neurogenic thoracic outlet syndromes: a comparison of true and nonspecific syndromes after surgical treatment. Surg Neurol 2006; 65:262-271.

3. Lee AD, Agarwal S, Sadhu D. Doppler Adson's test: predictor of outcome of surgery in non-specific thoracic outlet syndrome. World / Surg 2006; 30:291292.

4. Sheth RN, Campbell JN. Surgical treatment of thoracic outlet syndrome: a randomized trial comparing two operations. J Neurosurg Spine 2005; 3:355363.

5. Altobelli GG, Kudo T, Haas BT, Chandra FA, Moy JL, Ahn SS. Thoracic outlet syndrome: pattern of clinical success after operative decompression. I Vasc Surg 2005; 42:122-128.

6. Degeorges R, Reynaud C, Becquemin JP. Thoracic outlet syndrome surgery: longterm functional results. Ann Vasc Surg 2004;18:558-565.

7. Atasoy E. Combined surgical treatment of thoracic outlet syndrome: transaxillary first rib resection and transcervical scalenectomy. Hand Clin 2004; 20:7182, vii.

8. Balci AE, Balci TA, Cakir O, Eren S, Eren MN. Surgical treatment of thoracic outlet syndrome: effect and results of surgery. Ann Thorac Surg 2003; 75:10911096.

9. Axelrod DA, Proctor MC, Geisser ME, Roth RS, Greenfield LJ. Outcomes after surgery for thoracic outlet syndrome. I Vasc Surg 2001; 33:1220-1225.

10. Jordan SE, Ahn SS, Freischlag JA, Gelabert HA, Machleder HI. Selective botulinum chemodenervation of the scalene muscles for treatment of neurogenic thoracic outlet syndrome. Ann Vasc Surg 2000; 14:365-369.

11. Trief PM, Ploutz-Snyder R, Fredrickson $B E$. Emotional health predicts pain and function after fusion: a prospective multicenter study. Spine 2006; 31:823-830.

12. Scuderi GJ, Sherman AL, Brusovanik GV, Pahl MA, Vaccaro AR. Symptomatic cervical disc herniation following a motor vehicle collision: return to work comparative study of workers' compensation versus personal injury insurance status. Spine J 2005; 5:639-644.

13. Hagberg M. Clinical assessment, prognosis and return to work with reference to work related neck and upper limb disorders. G Ital Med Lav Ergon 2005; 27:51-57.

14. Balk ML, Hagberg WC, Buterbaugh GA, Imbriglia JE. Outcome of surgery for lateral epicondylitis (tennis elbow): effect of worker's compensation. Am J Orthop 2005; 34:122-126.

15. Osti OL, Gun RT, Abraham G, Pratt NL, Eckerwall G, Nakamura H. Potential risk factors for prolonged recovery following whiplash injury. Eur Spine J 2005; 14:90-94.

16. Saleh K, Nelson C, Kassim R, Yoon P, Haas S. Total knee arthroplasty in patients on workers' compensation: a matched cohort study with an average follow-up of 4.5 years. J Arthroplasty 2004; 19:310-312.

17. Goldberg EJ, Singh K, Van U, Garretson $\mathrm{R}$, An HS. Comparing outcomes of anterior cervical discectomy and fusion in workman's versus non-workman's compensation population. Spine / 2002; 2:408-414.

18. Marx RG, Jones EC, Angel M, Wickiewicz TL, Warren RF. Beliefs and attitudes of members of the American Academy of Orthopaedic Surgeons regarding the treatment of anterior cruciate ligament injury. Arthroscopy 2003;19:762-770.

19. Blyth FM, March LM, Nicholas MK, Cousins MJ. Chronic pain, work performance and litigation. Pain 2003; 103:41-47.

20. Nicholson GP. Arthroscopic acromioplasty: a comparison between workers' compensation and non-workers' compensation populations. J Bone Joint Surg Am 2003; 85-A:682-689.

21. Suter PB. Employment and litigation: improved by work, assisted by verdict. Pain 2002;100:249-257.

22. Hee HT III, Whitecloud TS, Myers L, Roesch W, Ricciardi JE. Do worker's compensation patients with neck pain have lower SF-36 scores? Eur Spine J 2002; 11:375-381.

23. Teasell RW. Compensation and chronic pain. Clin J Pain 2001; 17 (4 Suppl):S46S64.

24. Hawkins RJ, Plancher KD, Saddemi SR, Brezenoff LS, Moor JT. Arthroscopic subacromial decompression. I Shoulder Elbow Surg 200; 10:225-230.

25. Hodges SD, Humphreys SC, Eck JC, Covington LA, Harrom H. Predicting fac- tors of successful recovery from lumbar spine surgery among workers' compensation patients. I Am Osteopath Assoc 2001; 101:78-83.

26. Trief PM, Ploutz-Snyder R, Fredrickson $\mathrm{BE}$. Emotional health predicts pain and function after fusion: a prospective multicenter study. Spine 2006;31:823830.

27. Trief PM, Grant W, Fredrickson B. A prospective study of psychological predictors of lumbar surgery outcome. Spine 2000; 25:2616-2621.

28. Demondion $X$, Vidal C, Herbinet $P$, Gautier C, Duquesnoy B, Cotten A. UItrasonographic assessment of arterial cross-sectional area in the thoracic outlet on postural maneuvers measured with power Doppler ultrasonography in both asymptomatic and symptomatic populations. J Ultrasound Med 2006; 25:217-224.

29. Gillard J, Perez-Cousin M, Hachulla E Remy J, Hurtevent JF, Vinckier L. Diagnosing thoracic outlet syndrome: contribution of provocative tests, ultrasonography, electrophysiology, and helical computed tomography in 48 patients. Joint Bone Spine 2001; 68:416424.

30. Wadhwani R, Chaubal N, Sukthankar R, Shroff M, Agarwala S. Color Doppler and duplex sonography in 5 patients with thoracic outlet syndrome. J Ultrasound Med 2001; 20:795-801.

31. Longley DG, Yedlicka JW, Molina EJ, Schwabacher S, Hunter DW, Letourneau JG. Thoracic outlet syndrome: evaluation of the subclavian vessels by color duplex sonography. $A J R A m$ J Roentgenol 1992; 158:623-630.

32. Stanton PE, Jr., Vo NM, Haley T, Shannon J, Evans J. Thoracic outlet syndrome: a comprehensive evaluation. Am Surg 1988; 54:129-133.

33. Jordan SE, Machleder HI. Diagnosis of thoracic outlet syndrome using electrophysiologically guided anterior scalene blocks. Ann Vasc Surg 1998; 12:260264.

34. Degeorges R, Reynaud C, Becquemin JP. Thoracic outlet syndrome surgery: long-term functional results. Ann Vasc Surg 2004; 18:558-565.

35. Axelrod DA, Proctor MC, Geisser ME, Roth RS, Greenfield LJ. Outcomes after surgery for thoracic outlet syndrome. J Vasc Surg 2001; 33:1220-1225.

36. Katz JN, Stirrat CR, Larson MG, Fossel AH, Eaton HM, Liang MH. A self-admin- 
Pain Physician: May 2007:10:441-452

istered hand symptom diagram for the diagnosis and epidemiologic study of carpal tunnel syndrome. J Rheumatol 1990; 17:1495-1498.

37. Gummesson C, Atroshi I, Ekdahl C. The disabilities of the arm, shoulder and hand (DASH) outcome questionnaire: longitudinal construct validity and measuring self-rated health change after surgery. BMC Musculoskelet Disord 2003; 16:4-11.
38. Gay RE, Amadio PC, Johnson JC. Comparative responsiveness of the disabilities of the arm, shoulder, and hand, the carpal tunnel questionnaire, and the SF-36 to clinical change after carpal tunnel release. J Hand Surg [Am] 2003; 28:250-254.

39. Wolfe F, Smythe HA, Yunus MB, Bennett RM, Bombardier C, Goldenberg
DL, Tugwell P, Campbell SM, Abeles M, Clark P. The American College of Rheumatology 1990 Criteria for the Classification of Fibromyalgia. Report of the Multicenter Criteria Committee. Arthritis Rheum 1990; 33:160-172.

40. Task Force on DSM-IV. DSM-IV-TR. American Psychiatric Association, Washington, D.C., 2000. 